tially the two following properties of $\gamma(Z, W)$ :

(a) $\gamma(Z, W)$ has at $Z=W$ a logarithmic pole;

(b) $\gamma(Z, W)$ is a symmetric harmonic function;

while the third and most restrictive property of $\gamma(Z, W)$, that is, that it vanishes on the boundary $C$ of $B$, was only used in the transformation of (21) into $\left(21^{\prime}\right)$. It might be more convenient for practical computations to use instead of $\gamma(Z, W)$ simply the singularity function $-\log |z-w|$ which satisfies both conditions (a) and (b). The representation of the Neumann function is in this case a little more complicated, but on the other hand one is saved the effort of computing the Green's function of the domain with respect to Laplace's equation.

Finally, we wish to point out that we dealt with a particular type of the partial differential equation (1) only for the sake of a simple representation of the method. We indicated in our previous paper the possible generalizations of the type of equation, and for all those partial differential equations obtained one may construct the Green and Neumann functions in an analogous way.

HARVARD UNIVERSITY

\title{
THE ISOPERIMETRIC PROBLEM OF BOLZA WITH FINITE SIDE CONDITIONS
}

J. ERNEST WILKINS, JR.

1. Introduction. In view of recent improvements in the theory of the problem of Bolza in the calculus of variations, both with respect to the necessary $[2,6]^{1}$ and the sufficient conditions $[3,4,5,7]$, it seems worthwhile to investigate certain problems which, although not immediate special cases of the problem of Bolza, can be transformed by simple means into such problems. A comparatively simple problem of this nature is that of finding necessary conditions and sufficient conditions in order that an arc

C:

$$
a_{h}, y_{i}(x)
$$$$
\left(h=1, \cdots, r ; i=1, \cdots, n ; x_{1} \leqq x \leqq x_{2}\right)
$$

will minimize an expression of the form

$$
I(C)=g(a)+\int_{x_{1}}^{x_{2}} f\left(a, x, y, y^{\prime}\right) d x
$$

Received by the editors April 3, 1947.

1 Numbers in brackets refer to the bibliography at the end of the paper. 
in the class of $\operatorname{arcs} C$ which satisfy

$$
\begin{array}{rrr}
x_{s}=x_{s}(a), & y_{i}\left(x_{s}\right)=y_{i s}(a) & (s=1,2), \\
\phi_{\sigma}\left(a, x, y, y^{\prime}\right)=0 & (\sigma=q+1, \cdots, q+m<n), \\
g_{\rho}(a)+\int_{x_{1}}^{x_{2}} f_{\rho}\left(a, x, y, y^{\prime}\right) d x=0 & (\rho=q+1, \cdots, q+p),
\end{array}
$$

and in addition finite side conditions of the form

$$
\psi_{\mu}(a, x, y)=0 \quad(\mu=1, \cdots, q) .
$$

It is assumed that the functions appearing in equations (1.1) to (1.5) are continuous and have continuous partial derivatives of the first three orders in a region $R$ of points $\left(a, x, y, y^{\prime}\right)$. We suppose further that $x_{1}(a)<x_{2}(a)$ and that the matrix

$$
\left\|\begin{array}{c}
\phi_{\sigma y_{i}^{\prime}} \\
\psi_{\mu y_{i}}
\end{array}\right\|
$$

has rank $m+q$ on $R$.

For the case in which the parameters $a_{h}$ are fixed and the conditions (1.3) and (1.4) are absent, Bower [1] has given a treatment of this problem. ${ }^{2}$ Her treatment, however, is complicated by the fact that the associated isoperimetric problem of Bolza to be introduced in the next section is abnormal. Since normality is no longer needed as a hypothesis in either the necessary or the sufficient conditions, we can give a much simpler treatment of her problem. Introducing the parameters $a_{h}$ and the conditions (1.3) and (1.4) provides no essential difficulty.

2. Reduction to an equivalent isoperimetric problem of Bolza. Suppose that $C$ is an arc which satisfies (1.2) and (1.5). Then $C$ also satisfies

$$
\begin{aligned}
\phi_{\mu}\left(a, x, y, y^{\prime}\right) & \equiv \psi_{\mu x}(a, x, y)+\psi_{\mu y_{i}}(a, x, y) y_{i}^{\prime}=0, \\
g_{\mu s}(a) & \equiv \psi_{\mu}\left[a, x_{s}(a), y_{s}(a)\right]=0 \quad \text { when } s=1 .
\end{aligned}
$$

Conversely, if $C$ satisfies (1.2), (2.1) and (2.2), then it satisfies (1.5). Therefore, $C$ furnishes a weak or strong relative minimum for $I(C)$ in the class of arcs satisfying (1.2), (1.3), (1.4) and (1.5) if and only if $C$ furnishes a weak or strong relative minimum for $I(C)$ in the class of arcs satisfying (1.2), (1.3), (1.4), (2.1) and (2.2). Since conditions (2.1) are ordinary differential equations, and since conditions (2.2)

${ }^{2}$ This paper also contains references to the earlier work of Mayer, Bolza, Hadamard and Bliss. 
may be regarded as additional isoperimetric conditions, this second problem is an ordinary isoperimetric problem of Bolza.

3. The necessary conditions. Suppose that $C$ is an arc of class $C^{\prime \prime}$ which minimizes $I(C)$ in the class of arcs satisfying (1.2), (1.3), (1.4) and (1.5). Then we can prove that for each variation

$\gamma:$

$$
\alpha_{h}, \quad \eta_{i}(x)
$$

which satisfies

$$
\begin{gathered}
\eta_{i}\left(x_{s}\right)=\left[y_{i s h}-y_{i}^{\prime}\left(x_{s}\right) x_{s h}\right] \alpha_{h}, \\
\phi_{\sigma a_{h}} \alpha_{h}+\phi_{\sigma y_{i}} \eta_{i}+\phi_{\sigma y_{i}^{\prime} \eta_{i}^{\prime}}=0, \\
\psi_{\mu a_{h}} \alpha_{h}+\psi_{\mu y_{i}} \eta_{i}=0, \\
c_{\rho h} \alpha_{h}+\int_{x_{1}}^{x_{2}}\left(f_{\rho a_{h}} \alpha_{h}+f_{\rho y_{i}} \eta_{i}+f_{\rho y_{i}^{\prime} \eta_{i}^{\prime}}\right) d x=0,
\end{gathered}
$$

in which $c_{\rho h}$ is defined as

$$
c_{\rho h}=g_{\rho a_{h}}+\left.f_{\rho} x_{s h}\right|_{s=1} ^{s=2},
$$

and $x_{s h}=x_{s a_{h}}, y_{i s h}=y_{i s a_{h}}$, there exist constants $l_{0} \geqq 0, l_{\rho}, l_{\mu s}$, continuously differentiable functions $m_{\sigma}(x)$, and continuous functions $\lambda_{\mu}(x)$ which do not all vanish simultaneously at any point $x$, such that if

$$
\begin{aligned}
F\left(a, x, y, y^{\prime}, l, m, \lambda\right) & \equiv l_{0} f+l_{\rho} f_{\rho}+m_{\sigma}(x) \phi_{\sigma}+\lambda_{\mu}(x) \psi_{\mu}, \\
G(a, l) & \equiv l_{0} g+l_{\rho} g_{\rho}+l_{\mu s} g_{\mu s},
\end{aligned}
$$

then the following conditions, in addition to (1.2), (1.3), (1.4) and (1.5), hold along $C$ :

$$
\begin{gathered}
\frac{d}{d x} F_{y_{i}^{\prime}}=F_{y_{i}} \\
G_{a_{h}}+\left[\left(F-y_{i}^{\prime} F_{y_{i}^{\prime}}\right) x_{s h}+F_{y_{i}^{\prime} y_{i s h}}\right]_{s=1}^{s=2}+\int_{x_{1}}^{x_{2}} F_{a_{h}} d x=0 \\
E\left(a, x, y, y^{\prime}, Y^{\prime}, l, m, \lambda\right) \\
\equiv F\left(a, x, y, Y^{\prime}, l, m, \lambda\right)-F\left(a, x, y, y^{\prime}, l, m, \lambda\right) \\
-\left(Y_{i}^{\prime}-y_{i}^{\prime}\right) F_{y_{i}^{\prime}}\left(a, x, y, y^{\prime}, l, m, \lambda\right) \geqq 0
\end{gathered}
$$

whenever $\left(a, x, y, Y^{\prime}\right) \neq\left(a, x, y, y^{\prime}\right)$ and satisfies (1.3) and (2.1);

$$
F_{y_{i}^{\prime} y_{j}^{\prime}}\left(a, x, y, y^{\prime}, l, m, \lambda\right) \pi_{i} \pi_{j} \geqq 0
$$


whenever $\pi \neq 0$ and is such that

$$
\begin{aligned}
& \phi_{\sigma y_{i}^{\prime}}\left(a, x, y, y^{\prime}\right) \pi_{i}=0, \quad \psi_{\mu y_{i}}(a, x, y) \pi_{i}=0 \\
& J_{2}(\gamma) \equiv b_{h k} \alpha_{h} \alpha_{k}+\int_{x_{1}}^{x_{2}} 2 \omega\left(\alpha, x, \eta, \eta^{\prime}\right) d x \geqq 0
\end{aligned}
$$

where

$$
\begin{aligned}
& b_{h k}=G_{a_{h} a_{k}}+\left[\left(F-y_{i}^{\prime} F_{y_{i}^{\prime}}\right) x_{s h k}+F_{y_{i}^{\prime} y_{i s h k}}+F_{a k} x_{s h}+F_{a_{h}} x_{s k}\right. \\
& \left.+\left(F_{x}-y_{i}^{\prime} F_{y_{i}}\right) x_{s h} x_{s k}+F_{y_{i}}\left(x_{s h} y_{i s k}+x_{s k} y_{i s h}\right)\right]_{s=1}^{s=2}, \\
& 2 \omega=F_{y_{i} y_{j} \eta_{i} \eta_{j}}+2 F_{y_{i} y_{j}^{\prime} \eta_{i} \eta_{j}^{\prime}}+F_{y_{i}^{\prime} y_{j}^{\prime} \eta_{i}^{\prime} \eta_{j}^{\prime}}+2 F_{y_{i} a_{k}} \eta_{i} \alpha_{h} \\
& +2 F_{y_{i}^{\prime} a_{h}} \eta_{i}^{\prime} \alpha_{h}+F_{a_{h} a_{k}} \alpha_{h} \alpha_{k} \text {. }
\end{aligned}
$$

In fact, it is known [2] that if $C$ is of class $D^{\prime}$ and if $\gamma$ satisfies (3.1), (3.2), (3.4) and

$$
\begin{gathered}
\phi_{\mu a_{h}} \alpha_{h}+\phi_{\mu y_{i}} \eta_{i}+\phi_{\mu y_{i}^{\prime} \eta_{i}^{\prime}}=0, \\
g_{\mu 1 a_{h} \alpha_{h}}=0,
\end{gathered}
$$

then ${ }^{3}$ there exist continuous multipliers $l_{0} \geqq 0, l_{\rho}, l_{\mu}, m_{\sigma}(x), m_{\mu}(x)$, which do not all vanish simultaneously at any point $x$, such that if

$$
F_{0}=l_{0} f+l_{\rho} f_{\rho}+m_{\sigma} \phi_{\sigma}-m_{\mu} \phi_{\mu}, \quad G_{0}=l_{0} g+l_{\rho} g_{\rho}+l_{\mu} g_{\mu 1},
$$

then conditions (3.8), (3.9), (3.10) and (3.12) hold when $F$ and $G$ are replaced by $F_{0}$ and $G_{0}$. Moreover, we also have

$$
F_{0 y_{i}^{\prime}}=\int_{x_{1}}^{x} F_{0 y_{i}} d x+c_{i}
$$

for some constants $c_{i}$. An examination of the proof of this multiplier rule shows that if $C$ is known to be of class $C^{\prime \prime}$, then the multipliers $m_{\sigma}(x), m_{\mu}(x)$ are of class $C^{\prime}$. Furthermore, (3.16) can be written in the form (3.7) with $F$ replaced by $F_{0}$. If we now define $\lambda_{\mu}(x)$ and $l_{\mu s}$ by the equations

$$
\lambda_{\mu}(x)=m_{\mu}^{\prime}(x), \quad l_{\mu 1}=l_{\mu}+m_{\mu}\left(x_{1}\right), \quad l_{\mu 2}=-m_{\mu}\left(x_{2}\right),
$$

then the multipliers $l_{0}, l_{\rho}, l_{\mu s}, m_{\sigma}(x), \lambda_{\mu}(x)$ have the differentiability and continuity properties which they were asserted to have, and

${ }^{3}$ Cox derives these conditions for the nonisoperimetric problem. But the derivation of the necessary conditions for the isoperimetric problem from those for the nonisoperimetric problem is well known. 
they vanish simultaneously at some point $x$ if and only if the multipliers $l_{0}, l_{\rho}, l_{\mu}, m_{\sigma}(x), m_{\mu}(x)$ vanish simultaneously at some point $x$. If $F$ and $G$ are defined by (3.5) and (3.6) with these values of the multipliers, it is easy to see that conditions (3.7), (3.8), (3.9), (3.10) and (3.12) hold as stated when they hold for $F_{0}$ and $G_{0}$. Finally we observe that when $C$ satisfies (1.2) and (1.5) and $\gamma$ satisfies (3.1), then $\gamma$ satisfies (3.13) and (3.14) if and only if $\gamma$ satisfies (3.3).

4. The sufficiency theorems. These are now immediate consequences of the sufficiency theorems for the isoperimetric problem of Bolza $[5,7]$. If for every nonidentically vanishing variation which satisfies (3.1), (3.2), (3.3) and (3.4) there exist multipliers $(l, m, \lambda)$ satisfying the differentiability and continuity properties mentioned in $\$ 3$ such that equations (3.7) and (3.8) hold, and for which

$$
F_{y_{i}^{\prime} y_{j}^{\prime} \pi_{i} \pi_{j}}>0, \quad J_{2}(\gamma)>0
$$

whenever $\pi \neq 0$ and is such that equations (3.11) hold, then [7] $C$ gives $I(C)$ a proper weak relative minimum in the class of arcs satisfying (1.2), (1.3), (1.4) and (1.5). If in addition to (4.1) we require that (3.9) hold whenever the point $\left(a, x, y, y^{\prime}, l, m, \lambda\right)$ is in a neighborhood of those belonging to $C$ ( $l_{0}$ being held fast) and $\left(a, x, y, Y^{\prime}\right)$ satisfies (1.3) and (2.1), then [5] $C$ gives $I(C)$ a proper strong relative minimum in the class of arcs satisfying (1.2), (1.3), (1.4) and (1.5).

\section{BIBLIOGRAPHY}

1. J. W. Bower, The problem of Lagrange with finite side conditions, Contributions to the Calculus of Variations, 1933-1937, University of Chicago Press.

2. M. J. Cox, On necessary conditions for relative minima, Amer. J. Math. vol. 66 (1944) pp. 170-198.

3. M. R. Hestenes, The Weierstrass E-function in the calculus of variations, Trans. Amer. Math. Soc. vol. 60 (1946) pp. 51-71.

4. - - Theorem of Lindeberg in the calculus of variations, ibid. pp. 72-92.

5. - Sufficient conditions for the isoperimetric problem of Bolza in the calculus of variations, ibid. pp. 93-118.

6. E. J. McShane, Multipliers for Lagrange problems, Amer. J. Math. vol. 61 (1939) pp. 809-819.

7. - Sufficient conditions for a weak relative minimum in the problem of Bolza, Trans. Amer. Math. Soc. vol. 52 (1942) pp. 344-379.

American Optical Company 